\title{
Extinction of a Classically Conditioned Response: Red Nucleus and Interpositus
}

\author{
Karla Robleto and Richard F. Thompson \\ University of Southern California, Los Angeles, California 90089
}

It is well established that the cerebellum and its associated circuitry are essential for classical conditioning of the eyeblink response and other discrete motor responses (e.g., limb flexion, head turn, etc.) learned with an aversive unconditioned stimulus. However, brain mechanisms underlying extinction of these responses are still relatively unclear. Behavioral studies have demonstrated extinction to be an active learning process distinct from acquisition. Accordingly, this current understanding of extinction has guided neural studies that have tried to identify possible brain structures that could support this new learning. However, whether extinction engages the same brain sites necessary for acquisition is not yet clear. This poses an overriding problem for understanding brain mechanisms necessary for extinction because such analysis cannot be done without first identifying brain sites and pathways involved in this phenomenon. Equally elusive is the validity of a behavioral theory of extinction that can account for the properties of extinction. In this study, we looked at the involvement of the interpositus and the red nucleus in extinction. Results show that, although inactivation of both nuclei blocks response expression, only inactivation of the interpositus has a detrimental effect on extinction. Moreover, this detrimental effect was completely removed when inactivation of the interpositus was paired with electrical stimulation of the red nucleus. These findings speak to the important role of cerebellar structures in the extinction of discrete motor responses and provide important insight as to the validity of a particular theory of extinction.

Key words: extinction; cerebellum; classical conditioning; learning; eyeblink; red nucleus; interpositus; inferior olive

\section{Introduction}

A great deal more is known concerning the neural substrates for acquisition of classical conditioning of eyeblink and other discrete responses (Thompson and Krupa, 1994; Yeo and Hesslow, 1998; Nores et al., 2000; Steinmetz, 2000; Christian and Thompson, 2003) than about the neural substrates of extinction (Bouton, 2004; Mauk and Ohyama, 2004; Rescorla, 2004; Robleto et al., 2004). It has been clear since Pavlov's studies that extinction is not merely the fading away of memory, namely, spontaneous recovery, disinhibition, rapid reacquisition, etc. The general explanation of these phenomena at the behavioral level is that extinction involves new learning that somehow interferes with performance of the conditioned response (CR).

A number of behavioral theories have been proposed to account for this interference (Poulos et al., 2006), among the most prominent being the response-extinction hypothesis (Rescorla, 1997) and the context hypothesis (Bouton and Nelson, 1994; Kehoe et al., 2004). More recently, data using genetic and pharmacological techniques argue for the existence of a dissociation between response expression and extinction (Thomas et al., 1998; Ouyang and Thomas, 2005) in which memory re-

Received July 11, 2007; revised Dec. 21, 2007; accepted Jan. 19, 2008

This work was supported in part by National Science Foundation Grant IBN-9215069, National Institutes of Health Grant AG0145, and the University of Southern California. We thank Andrew Poulos for facilitating microscopy work.

Correspondence should be addressed to Karla Robleto, University of Southern California, 3641 Watt Way, HNB522, Los Angeles, CA 90089. E-mail: krobleto@usc.edu.

DOI:10.1523/JNEUROSCI.4781-07.2008

Copyright $\odot 2008$ Society for Neuroscience $\quad$ 0270-6474/08/282651-08\$15.00/0 trieval and not conditioned responding is critical in extinction. Also supporting dissociation of CR expression and extinction are data using olfactory conditioning in Drosophila showing that blocking conditioned responding by inactivation of specific cells in the olfactory pathway does not prevent extinction (Schwaerzel et al., 2002).

The immediate goal of the present experiment is to determine the extent to which the neuronal substrates critically involved in acquisition of the eyeblink CR are also critical for extinction of the $\mathrm{CR}$ and the implications of these results for theories of extinction. Extensive data have shown that reversible inactivation of the cerebellar interpositus nucleus (IN) completely prevents acquisition of the eyeblink CR (Krupa et al., 1993; Hardiman et al., 1996; Krupa and Thompson, 1997) and also appears to prevent extinction of the CR (Hardiman et al., 1996; Ramnani and Yeo, 1996). However, inactivation of motor nuclei (MN), which does not interfere with acquisition of the $\mathrm{CR}$, although of course completely preventing performance of the $\mathrm{CR}$, also prevents extinction of the CR (Krupa et al., 1996; Krupa and Thompson, 2003). This would seem to support the response theory of extinction, i.e., inactivation of the IN could prevent extinction because it prevents occurrence of the behavioral CR. Here we use eyeblink conditioning along with pharmacological and electrophysiological approaches to test the idea that extinction depends on mechanisms that require performance of the conditioned response. Evidence to be presented argues against the response theory of extinction and is consistent with the view that the inferior olive (IO) is the "teaching signal" for extinction. 


\section{Materials and Methods \\ Experiment 1: reversible inactivations of the interpositus nucleus}

Studies using cerebellar aspirations or muscimol microinfusions have reported that inactivation of the cerebellum prevents extinction of the eyeblink CR (Perrett and Mauk, 1995; Ramnani and Yeo, 1996). However, the exact involvement of specific cerebellar regions is difficult to determine from such data given the inconsistent extent of the lesions or the uncertain spread of the infusions in the targeted area. Here we infuse much lower volumes of muscimol $(0.1 \mu \mathrm{l})$ than the $1 \mu \mathrm{l}$ used by Ramnani and Yeo (1996) to achieve a more targeted inactivation of the IN. Highly localized effects using such low infusion volumes in the IN are consistent with previous observations using autoradiographic techniques (Krupa, 1993). Such data indicate maximal spread of the drug to be predominantly confined to the IN when volumes of $<110 \mathrm{nl}$ are infused.

\section{Subjects}

Twenty New Zealand White rabbits weighing between 2.2 and $3.0 \mathrm{~kg}$ at the time of surgery were used in the experiment. Animals were housed individually and maintained on a $12 \mathrm{~h}$ light/dark cycle with ad libitum access to water and food. Rabbits were divided into three groups. One group received infusions of muscimol that targeted the IN consisting of 1 $\mu \mathrm{l}(0.01 \mathrm{M}$ solution $)$. The second group received muscimol infusions into the IN consisting of $0.1 \mu \mathrm{l}(0.01 \mathrm{M})$, a much smaller dose that was less likely to spread to the cortex or HVI (hemispheric lobule VI) (Krupa et al., 1993), ruling out confounding effects of cortical regions. The final group received infusions of saline into the IN.

\section{Surgery}

All animals were anesthetized with subcutaneous injections of ketamine $(60 \mathrm{mg} / \mathrm{kg})$ and xylazine $(8 \mathrm{mg} / \mathrm{kg})$ and placed on a stereotaxic frame with a constant flow of $1.5-2 \%$ of halothane in oxygen throughout the length of the surgical procedure. Under aseptic surgical conditions, an anterior to posterior incision was made in the scalp to retract the skin and periostium and expose the skull. A small hole of $\sim 1.5 \mathrm{~mm}$ in diameter was drilled through the bone at the anterior/posterior and lateral/medial coordinates of the target structure. A custom-made guide cannula (26 gauge) $25 \mathrm{~mm}$ in length was implanted into the target nuclei with bregma $1.5 \mathrm{~mm}$ dorsal to lambda. The guide cannula was lowered with a stainless steel stylet (Elephant brand 000 insect pin) inserted and extending 1.5 $\mathrm{mm}$ below the tip. The outer cannula was sharpened to minimize damage to brain tissue during its implantation. This cannula assembly was lowered slowly with a stereotaxic manipulator that targeted the following coordinates based on the stereotaxic atlas of McBride and Klemm (1968): $0.7 \mathrm{~mm}$ anterior, $5.1 \mathrm{~mm}$ lateral, and $14.5 \mathrm{~mm}$ ventral. Subsequently, four holes were drilled to fit surgical stainless steel screws that anchored a head stage constructed from dental acrylic (Harry J. Bosworth, Skokie, IL). A Plexiglas mount built to hold a minitorque potentiometer (Subminiature Instruments, Ogden, UT) during behavioral training was also cemented to the head stage. Skin surrounding the dental acrylic was aseptically cleaned with antibiotic ointment (iodine). Animals were allowed to recover for a period of 1 week before the beginning of behavioral training. During the first 2 postoperative days, animals were given two daily injections of analgesic (buprenorphine hydrochloride) and were monitored throughout the length of their recovery. All procedures and animal care were approved by the University of Southern California Institutional Animal Care and Use Committee in accordance with $\mathrm{Na}-$ tional Institutes of Health guidelines.

\section{Apparatus}

Animals were habituated and trained in a padded Plexiglas restraint and placed into a behavioral recording chamber. During training, nictitating membrane (NM) movements were measured with a minitorque potentiometer temporarily attached to the animal's head stage. Voltage changes attributable to movement of the NM were measured via mechanical movement of a bar attached to the potentiometer and connected by a thread lead hook to a suture loop implanted in the left NM. The voltage signal was stored and recorded on a computer using custom software written in Forth (Lavond and Steinmetz, 1989). The software also controlled delivery of the stimuli during training. Data were subsequently analyzed off-line. Infusions were delivered to the targeted structure using 1 and $10 \mu \mathrm{l}$ syringes (Hamilton Instruments, Reno, NV) for infusions of a volume of 0.1 and $1 \mu \mathrm{l}$, respectively. Syringes were connected to a polyethylene tube (A-M Systems, Carlsborg, WA) attached to an injection cannula (31 gauge) $26.5 \mathrm{~mm}$ in length. Drug was delivered by compression of the syringe using an infusion pump (Harvard Apparatus, Holliston, MA) at a rate of $0.38 \mu \mathrm{l} / \mathrm{min}$.

\section{Behavioral training procedures}

Phase I. Animals were presented with four daily sessions of tone-air puff pairings. Each acquisition session consisted of 100 trials divided into 10 blocks of 10 trials. Each block contained one tone-alone trial, one air puff-alone trial, and eight tone-air puff pairings. Intertrial intervals ranged randomly between 20 and $40 \mathrm{~s}$. Tone-air puff pairings consisted of a tone conditioned stimulus (CS) presented for $350 \mathrm{~ms}(1 \mathrm{kHz}, 85 \mathrm{~dB})$ with a coterminating corneal air puff unconditioned stimulus (US) (100 $\mathrm{ms}, 3 \mathrm{psi}$ ). CRs were defined as nictitating membrane extensions of at least $0.5 \mathrm{~mm}$ occurring between 35 and $250 \mathrm{~ms}$ after CS onset. On tonealone trials, the critical CR range was between 35 and $750 \mathrm{~ms}$ after CS onset. In phase I, rabbits were required to meet the following criteria: (1) perform at least eight conditioned responses within nine consecutive trials by the end of the third session, and (2) give at least $80 \%$ conditioned responses during the final acquisition session. This ensured that all rabbits had reached the same level of performance by the end of the fourth session. At the end of this phase, animals were randomly assigned to either a control or an experimental group.

Phase II. After acquisition, animals received four daily sessions of tonealone training. One hour before the beginning of each training session, rabbits received infusions of muscimol or saline targeting the structure of interest. Infusions were administered by removal of the inner stylet and insertion through the guide cannula of the injection cannula. Delivery of muscimol was followed by a waiting period of $3 \mathrm{~min}$ to ensure absorption of the drug into the brain before the infusion cannula was removed and replaced with the stylet.

Phase III. Extinction training without drug infusions were given to rabbits for 4 consecutive days or until CR levels dropped to $15 \%$ or less.

Phase IV. To measure the ability of animals to reacquire the response, CS-US pairings were presented again to animals on the day after the conclusion of extinction training.

\section{Histology}

After training, an insulated electrode with $250 \mu \mathrm{m}$ of tip exposure was lowered through the guide cannula. A $100 \mu \mathrm{A}$ current was subsequently passed through the electrode for $10 \mathrm{~s}$ to create a marking lesion at the site of injection. Animals were then given an intravenous overdose of sodium pentobarbital and perfused intracardially with saline and $10 \%$ Formalin. Brains were then extracted and preserved for later histological reconstruction in $10 \%$ Formalin for a minimum of $3 \mathrm{~d}$. Brains were then embedded in an albumin and gelatin mixture, sectioned at $80 \mu \mathrm{m}$, and mounted for later staining. Mounted sections were Nissl stained with cresyl violet and counterstained with Prussian blue to label iron deposits left by the tip of the electrode at the time of the marking lesion. Injection sites were then recorded using a dissecting microscope (Fig. 1).

\section{Experiment 2: electrical stimulation of the red nucleus during interpositus inactivation}

In acquisition, reversible inactivation of CR output pathways, the IN, red nuclei (RN), and MN (Clark and Lavond, 1993; Krupa et al., 1993, 1996), cause complete abolition of CR expression, yet only lesions of the IN prevent learning of the original association (Krupa et al., 1993; Krupa and Thompson, 1997). Given the hypothesized role of response performance in extinction, it is critical to evaluate the effect of response performance during extinction training with inactivation of the IN. Here we address this problem by maintaining responding through brain stimulation of the RN during IN inactivations. The stimulation protocol used in this experiment is the same as that used by Chapman et al. (1988). Under these conditions, although eyeblinks are elicited, direct stimulation of the $\mathrm{RN}$ does not support conditioning. Other stronger stimulation protocols 
0.0

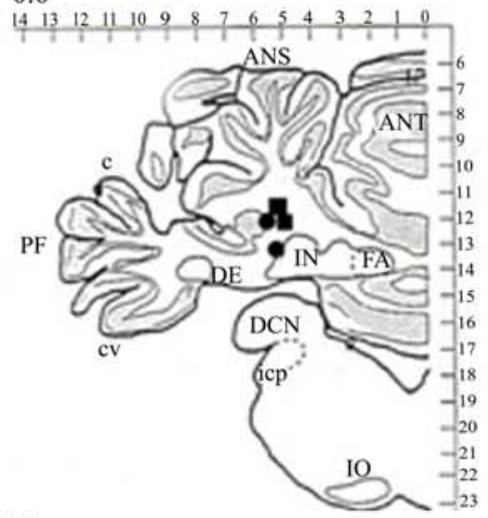

0.5

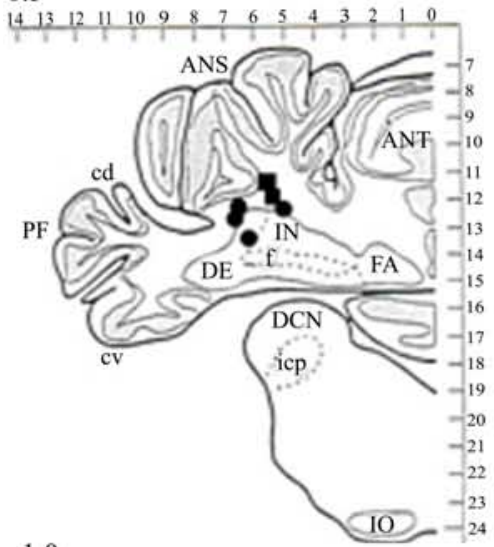

1.0

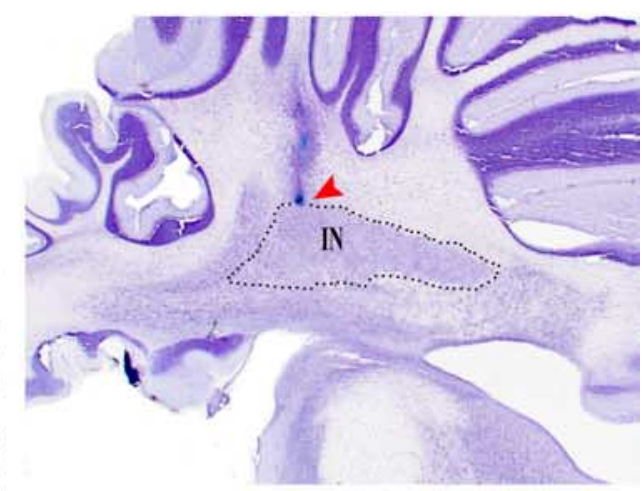

$14 \quad 13 \quad 12111098 \quad 8 \quad 65443210$
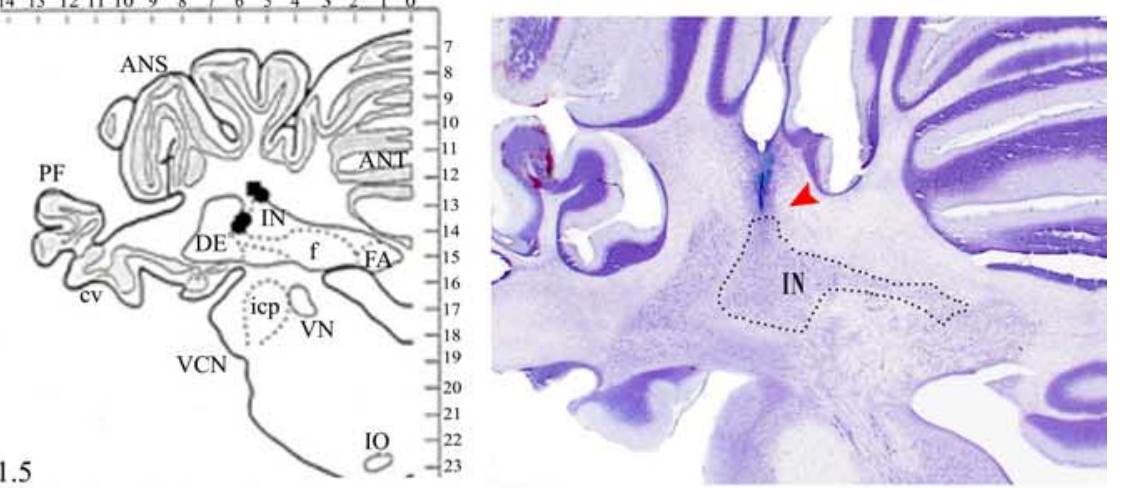

\begin{tabular}{lllllllllllll}
12 & 11 & 10 & 9 & 8 & 7 & 6 & 5 & 4 & 3 & 2 & 1 & 0 \\
\hline 1 & 1 & 1 & 1 & 1 & 1 & 1 & 1 & 1 & 1 & 1
\end{tabular}
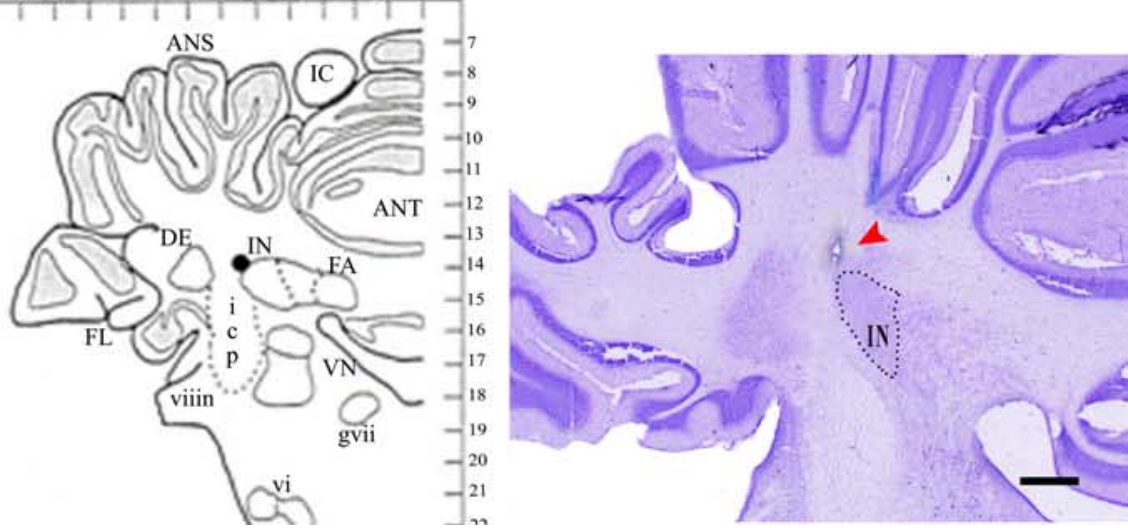

Figure 1. Histological reconstruction and representative micrographs of cannula placements targeting the IN. Circles, $1 \mu$ group; squares, $0.1 \mu \mathrm{l}$ group. Numerals above plates represent distance in millimeters rostral from lambda. IN, Interpositus nucleus; DE, dentate nucleus; FA, fastigial nucleus; ANS, ansiform lobule; ANT, anterior lobe; DCN, dorsal cochlear nucleus; I0, inferior olive; PF, paraflocculus; $c$, lobule c; cv, ventral crus; icp, inferior cerebellar peduncle; $c d$, dorsal crus; $f$, fibers; VCN, ventral cochlear; VN, vestibular nuclei; $F L$, flocculus; $I C$, inferior colliculus; vi, abducens nucleus; gvii, genu of the seventh nerve; viiin, nerve of the eight nucleus. Scale bar, $1000 \mu \mathrm{m}$.
(Nowak et al., 1997) have reported induction of learning and are not used here.

Subjects

Twenty-two New Zealand White rabbits weighing between 2.2 and $3.0 \mathrm{~kg}$ were implanted with a bipolar stimulating electrode targeting the RN and a cannula in the interpositus. Rabbits in the experimental group received infusions of muscimol that targeted the IN consisting of $0.1 \mu \mathrm{l}(0.01$ $\mathrm{M})$. Animals in the control group received infusions of saline.

\section{Surgery}

Surgical procedures were identical to those described in experiment 1 with the following exception: a set of bipolar electrodes was slowly implanted into the RN. The electrodes were custom designed and consisted of two number 000 stainless steel insect pins with several layers of epoxylite insulation and a tip exposure of 250-300 $\mu \mathrm{m}$. Electrodes were stereotaxically placed into the $\mathrm{RN}$ based on the following coordinates (from bregma): $8.5 \mathrm{~mm}$ posterior, $1.0 \mathrm{~mm}$ medial, and $15.5 \mathrm{~mm}$ ventral. Final placement of electrodes in all animals was determined on observable movement of the NM or eyelids when current was passed.

\section{Apparatus and behavioral}

training procedures

The training apparatus is identical to that described in Experiment 1. Electrical stimulation of the $\mathrm{RN}$ during inactivation of the IN was delivered using a Grass Instruments (Quincy, MA) stimulator and a constant-current isolation unit (Grass Instruments). Behavioral training was performed as described previously with the following exceptions.

Phase II. One hour after infusions were administered, animals were put inside each training chamber in which the stimulator was connected to the electrodes. Before the beginning of each training session, $\mathrm{RN}$ stimulation parameters were determined. Parameters for each animal varied slightly according to the minimum amount of current necessary to evoke a response. Intensities varied from 400 to $700 \mu \mathrm{A}$ and were delivered at a rate of $200 \mathrm{~Hz}$ with pulses of $0.2 \mathrm{~ms}$ in duration. Animals were only used if the stimulation successfully evoked an observable eyeblink. After threshold measurements, animals were then given a baseline tone-alone test to confirm the absence of response expression and thus successful inactivation of the IN. If responses were observed during this baseline trial, animals were brought back a few hours later and infusions were administered again. Failure of the infusions to suppress response expression this second time suggested an inaccurate cannula placement, and exclusion of these animals from the study followed. Rabbits that met stimulation and inactivation criteria were then given 100 trials of tonealone training $(1 \mathrm{kHz}, 85 \mathrm{~dB})$ during each extinction session (four extinction sessions in total). Stimulation timing during training was determined by taking the average response onset of six randomly selected well trained animals. This yielded an average onset of $165 \mathrm{~ms}$ after tone presentation. Based on this calculation, responses 
were induced by applying the stimulation at exactly this time during the trial.

Phase III. Extinction training without drug infusions or electrical brain stimulation was given to rabbits for 4 consecutive days or until CR levels dropped to $15 \%$ or less.

\section{Histology}

After training, a $100 \mu \mathrm{A}$ current was passed through the electrode and cannula for $10 \mathrm{~s}$ to create a marking lesion at the site of injection (Fig. 2).

\section{Experiment 3: inactivations of the red nucleus}

Subjects, surgeries, behavioral training, and histology

To further clarify whether observations from experiment 2 were attributable to experimental maintenance of the response during extinction, we proceeded to investigate the effects of $\mathrm{RN}$ inactivation during extinction. As a critical component of the CR pathway, inactivation of the RN completely abolishes expression of the CR yet does not interfere at all with learning of the eyeblink response (Clark and Lavond, 1993; Krupa et al., 1993).

Fourteen male New Zealand White rabbits weighing between 2.2 and $3.0 \mathrm{~kg}$ at the time of surgery were used in the experiment. Animals in the experimental group were infused with $0.1 \mu \mathrm{l}$ (0.01 M solution) of muscimol into the red nucleus. The control group was infused with saline. Cannula targeting the red nucleus was surgically implanted following the surgery protocol previously mentioned here. Coordinates used were based on the stereotaxic atlas of McBride and Klemm (from bregma): $8.5 \mathrm{~mm}$ posterior, 1.0 $\mathrm{mm}$ lateral, and $15.5 \mathrm{~mm}$ ventral. Postsurgery and training was followed using the same protocol described in experiment 1 . After training, cannula positions were determined by a marking lesion. All but two animals in the control group were included in the experiment for analyses. One animal failed to reach learning criteria and was thus excluded from additional training. A second animal had postsurgery complications and could not be included in the study. A final statistical analysis was done for seven animals in the experimental group and five in the control group.

\section{Results}

\section{Experiment 1}

Of the 20 animals that began the experiment, two in the group infused with $0.1 \mu \mathrm{l}$ of muscimol and one animal in the control group were not included in the analyses. One of the animals in the $0.1 \mu \mathrm{l}$ group developed a brain infection during the drug infusion phase of extinction training. The second rabbit showed high CR frequencies during IN inactivation sessions. Later histological examination confirmed misplacement of the cannula tip in the white matter dorsal to the IN and close to the paramedian lobe. The animal excluded in the control group failed to reach learning criteria during the acquisition phase. As a result, data shown are for seven animals in the $1 \mu \mathrm{l}$
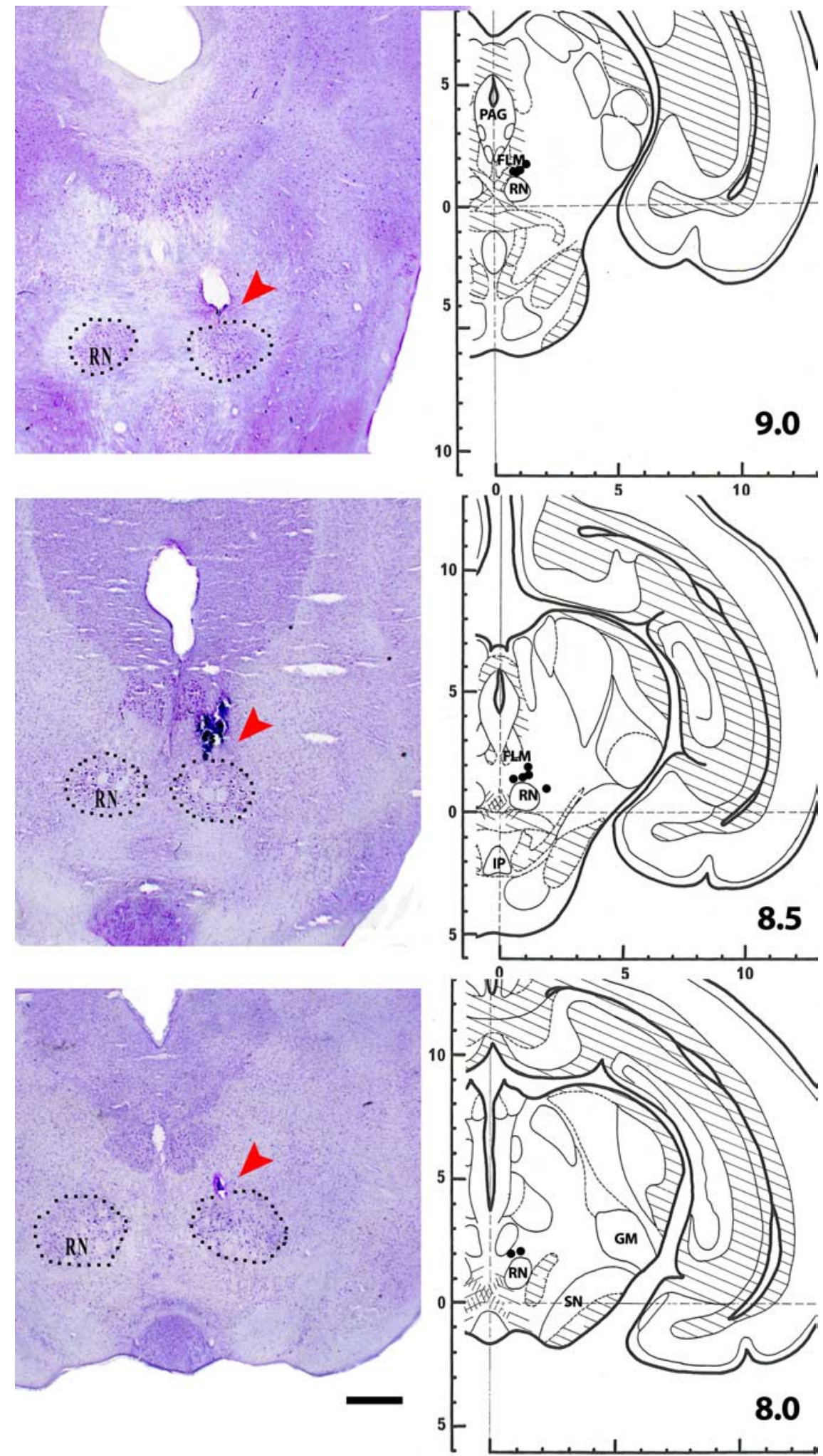

Figure 2. Histological reconstruction and representative micrographs of cannula/electrode placements targeting the red nucleus. Numerals in millimeters represent distances caudal to bregma. IP, Interpeduncular nucleus; GM, medial geniculate nucleus; PAG, periaqueductal gray; RN, red nucleus; SN, substantia nigra; FLM, fasciculi longitudinalis medialis. Scale bar, $1000 \mu \mathrm{m}$.

group, five rabbits in the $0.1 \mu \mathrm{l}$ group, and five in the control group.

Data show clearly that inactivations with muscimol of the IN completely block extinction (and expression) of the conditioned 


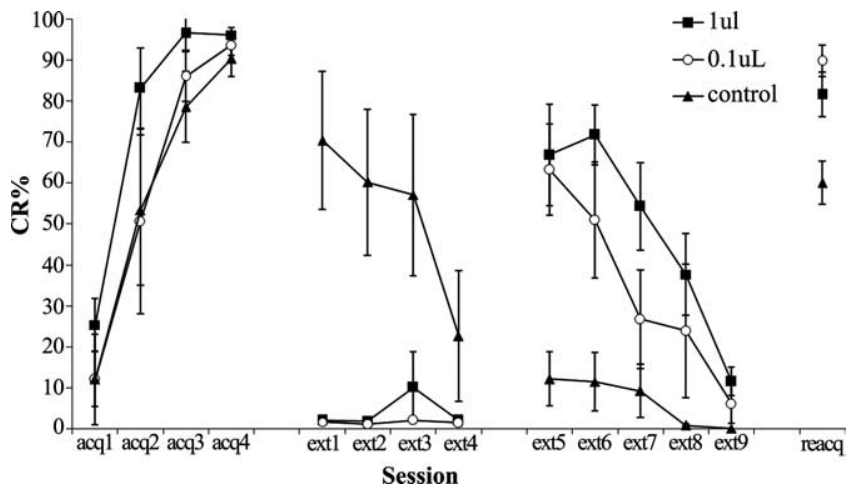

Figure 3. Mean \pm SEM percentage of conditioned responses for all three groups during each day of training. Animals in the experimental group received infusions of muscimol targeting the IN before extinction sessions $1-4$. Starting on day 5 of extinction, animals were trained without the drug treatment.

response. Subsequent postinfusion extinction training revealed that animals were able to extinguish at rates that were identical to those of the control group, indicating that the effects observed were not attributable to motor deficits or the result of damage to the nucleus because of repeated drug infusions. Moreover, experimental animals extinguished at the same rate as controls extinguished during the first days of extinction training. These results are entirely consistent with previous findings showing that inactivations of the cerebellum using higher doses of muscimol prevent extinction (Ramnani and Yeo, 1996).

Figure 3 shows the mean percentage CRs during each session of acquisition and extinction training for all three groups. Animals acquired the association and met learning criteria for phase I of the experiment with no overall significant differences in performance (average \pm SEM CR percentage on the last day of acquisition, $93.3 \pm 1.5 \%$ ). There were significant effects of days (sessions) consistent with increases in CR frequency indicative of acquisition of the association $\left(F_{(3,42)}=80.6 ; p<0.01\right)$. An ANOVA of day 3 found a significant main effect between the control group and the $1 \mu \mathrm{l}$ group $(p=0.042)$, but a repeatedmeasures ANOVA found no overall effect between subjects effects, indicating that all three groups attained similar CR rates before entering the extinction phase of the experiment $\left(F_{(2,14)}=\right.$ 2.23; $p=0.144)$.

A repeated-measures ANOVA of phase II revealed a significant main effect between the groups (extinction days $1-4)\left(F_{(2,14)}\right.$ $=16.01 ; p<0.01)$. A post hoc analyses found that differences were significant between controls and the $1 \mu$ l group $(p<0.01)$ as well as the $0.1 \mu$ lgroup $(p<0.01)$, demonstrating that infusions of muscimol targeting the interpositus nucleus effectively abolished the CR in both experimental groups and thus successfully inactivated the nucleus during extinction training. On average, the 1 and $0.1 \mu \mathrm{l}$ groups performed at levels of $3.9 \pm 6.2$ and $1.5 \pm 7.3 \%$ (mean \pm SEM), respectively, whereas controls displayed much higher CR frequencies (mean \pm SEM, $52.5 \pm 7.3 \%$ ). Nonetheless, CR frequencies for the control group decreased during each consecutive extinction day (mean \pm SEM; extinction 1, $70 \pm 8 \%$; extinction 2, $60 \pm 8.5 \%$; extinction $3,57 \pm 11.2 \%$ ) down to $22 \pm 7.6 \%$ on day 4 of extinction, indicating a gradual loss of response performance attributable to tone-alone training.

Analyses of CR performance during extinction training with no drug (phase III) revealed that subjects in the experimental groups showed significantly high CR frequencies during the first extinction session (mean \pm SEM; $1 \mu$ l group, $66.8 \pm 9.1 ; 0.1 \mu \mathrm{l}$ group, $63.2 \pm 10.8)$ compared with controls $\left(12.1 \pm 10.8 ;_{\mathrm{F}(2,14)}\right.$ $=8.5 ; p<0.004)$, who were already performing at almost baseline levels. There was a main group effect during this phase of training $\left(F_{(2,14)}=10.38 ; p<0.002\right)$, confirming that extinction levels of experimental subjects were different from controls. Although no differences were found between overall performances of both experimental groups ( $p<0.298$ ), there does seem to be a trend with subjects in the $0.1 \mu$ l group showing higher extinction levels than the $1 \mu$ l group. Experimental subjects extinguished the conditioned response during postinfusion tone-alone training (days 5-9) at rates that were identical to those of the control group during days $1-5$, as revealed by a repeated-measures $\operatorname{ANOVA}\left(F_{(1,14)}=0.70 ; p=0.511\right)$. These data suggest that IN inactivations completely prevent extinction from occurring, yet animals are able to extinguish at the same rate as controls once the inactivation is released.

Despite almost no production of CRs on the last day of extinction training, all groups were able to reacquire the response at levels that were different from naive training (mean \pm SEM; $77.1 \pm 2.7 \%)$. However, there was a significant group effect $\left(F_{(2,14)}=9.6 ; p<0.002\right)$. Controls displayed significantly lower levels of CRs compared with the $1 \mu$ l group $(p<0.014)$ and the $0.1 \mu$ l group $(p<0.002)$. No difference in CR reacquisition was observed between the experimental groups $(p<0.441)$. The fact that experimental animals achieved significantly higher CR levels suggests that these animals were only able to start extinguishing the response with the nucleus intact and were therefore "exposed" to less days of extinction training compared with controls, hence, more easily reacquiring the association.

\section{Experiment 2}

Here we have manipulated the response via brain stimulation of the $\mathrm{RN}$, an essential structure in the CR output pathway. Of the twentytwo rabbits that began the study, four in the experimental group and five in the control group were included for additional analyses. Animals excluded in the control group had postsurgery complications that prevented subsequent training. Rabbits not included in the experimental group either showed CRs during the baseline IN inactivation test or failed to exhibit an observable eyeblink via electrode stimulation. Also, several animals did not learn the CS-US association and were thus excluded early during training. Histological analyses of these animals showed cannula and/or electrode placements that penetrated most of the nuclei (IN and/or RN), suggesting lesioning of the critical region of the structure.

Data here demonstrate that stimulation of the RN completely reverses the blocking effects of IN inactivation during extinction (see experiment 1). Figure 4 shows the overall CR performance for both groups in the experiment. During the acquisition phase of the study, a significant day effect was found, consistent with increases in CR frequency indicative of learning of the association $\left(F_{(3,21)}=73.4 ; p<0.00\right)$. Furthermore, both the experimental and control group acquired the association at asymptotic levels (average CR percentage on the last day of acquisition: controls, $88.1 \pm 3.2 \%$; experimental, $90.3 \pm 3.6 \%$ ) with no significant differences in performance between the groups before entering phase II of the study $\left(F_{(1,7)}=1.6 ; p<0.249\right)$.

During phase II of extinction training (days 1-4), animals in the control group showed a gradual decrease of CRs attributable to tone-alone training (mean \pm SEM, $70.3 \pm 6.03 \%$ ), and, by the last day of this phase (day 4), the average CR performance for controls was down to $35 \pm 13.1 \%$. In contrast, experimental animals trained under IN inactivation paired with brain stimulation displayed high CR levels throughout this phase (mean \pm 


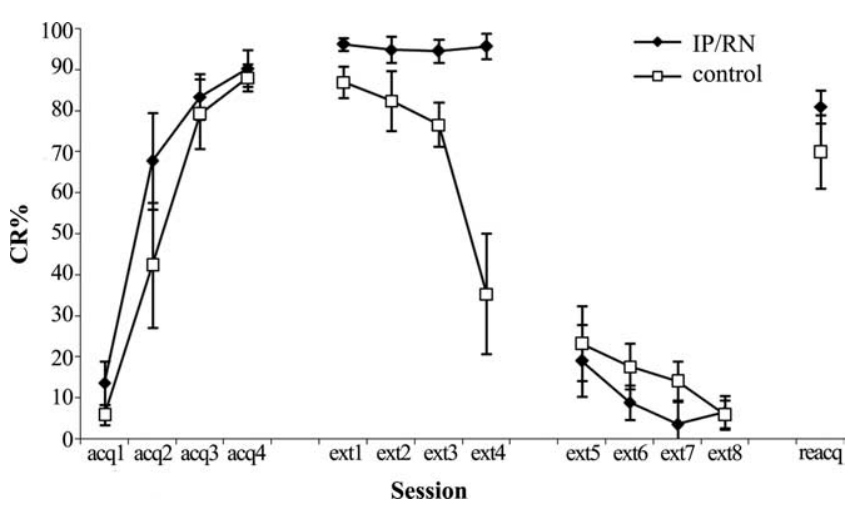

Figure 4. Mean \pm SEM percentage of conditioned responses for both groups during each day of training. Animals in the experimental group received infusions of muscimol targeting the interpenduncular nucleus (IP) paired with electrical brain stimulation to the RN on extinction sessions $1-4$. Starting on day 5 of extinction, animals were trained without the drug treatment and brain stimulation.

SEM, $95.4 \pm 1.5 \%$ ) up to day 4 (mean \pm SEM, $95.7 \pm 2.7 \%$ ). No significant day effects were found $\left(F_{(3,9)}=0.10 ; p<0.95\right)$, indicative of successful induction of the response via stimulation of the RN despite IN activity blockage. As would be expected, a main effect was found between controls and experimental groups during this phase of the study $\left(F_{(1,7)}=12.9 ; p<0.009\right)$.

Posttreatment extinction clearly showed that, despite IN inactivations, brain stimulation of the $\mathrm{RN}$ resulted in extinction rates that were statistically identical to those of controls. Analyses of CR performance after experimental manipulations were stopped (phase III) revealed that, during the first extinction session of this phase (day 5), animals in the experimental group showed a great loss in CR frequency (mean \pm SEM, $19 \pm 7.6 \%$ ) that was no different from controls $\left(23.2 \pm 7.6 \% ; F_{(1,7)}=0.13 ; p<0.72\right)$. Although there seems to be a trend of lower levels of performance for experimental subjects, there were no significant overall differences between the groups during this phase $\left(F_{(1,7)}=1.44 ; p<\right.$ $0.27)$. Moreover, just as for the control group, CR performance of experimental animals gradually decreased to almost baseline levels with additional tone-alone training, indicating that brain stimulation had no effect on the ability to extinguish the response. By the last day of extinction training, both groups were performing at almost baseline levels (mean \pm SEM; experimental, $6.5 \pm 3.5 \%$; controls, $5.8 \pm 3.1 \%$ ). Data from experimental animals further show that, when the CS-US association was reintroduced after extinction training, animals were able to reacquire the response at high levels just as controls. This is important because it demonstrates that the low response performance of experimental animals observed on days 5-8 was not attributable to damage of the $\mathrm{RN}$ caused by the applied stimulation.

\section{Experiment 3}

Inactivations with muscimol of the $\mathrm{RN}$ had no effect at all on the ability to extinguish the response, although infusions completely prevented expression of the response. Indeed, after inactivations were stopped, animals showed identical levels of extinction compared with controls, indicating that inactivation of the nucleus did not at all prevent extinction during treatment days. This effect was seen with very small doses of muscimol $(0.1 \mu \mathrm{l})$ that were very unlikely to diffuse to neighboring structures.

Figure 5 shows performance across training days for both the experimental and control groups. By day 4 of acquisition training, both groups were performing at asymptotic levels (mean \pm

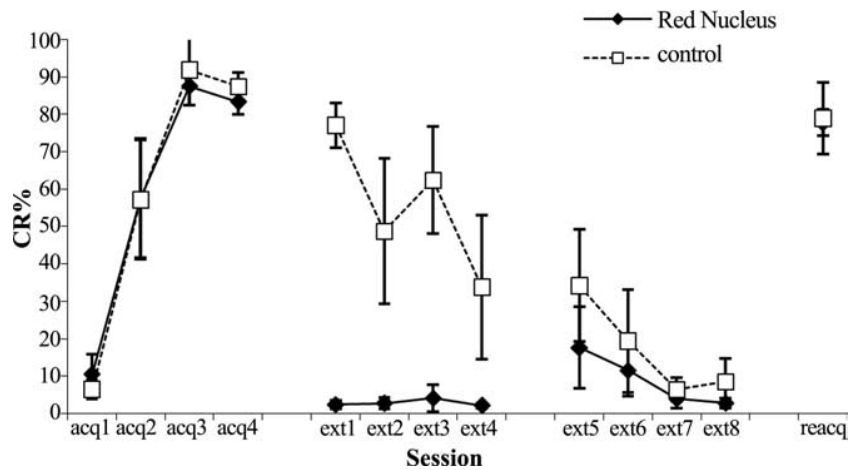

Figure 5. Mean \pm SEM CR percentages of groups during each training day. Animals in the experimental group showed no (R expression during muscimol infusions (ext1- ext4), confirming successful inactivation of the red nucleus. Infusions were stopped on day 5 of extinction training.

SEM, $85.4 \pm 2.3 \%)$. Also, both groups attained similar levels of learning before extinction training $\left(F_{(1,10)}=0.017 ; p=0.90\right)$. There was also a significant day effect, indicating learning during training $\left(F_{(3,30)}=48.2 ; p<0.00\right)$.

During phase II of the study, animals in the experimental group showed lower response frequencies (mean \pm SEM, $2.9 \pm$ $6.7 \%$ ) compared with controls $(54 \pm 7.9 \%)$. A main group effect was found during this phase of the study $\left(F_{(1,10)}=24.02 ; p<\right.$ $0.001)$, confirming that inactivations targeting the $\mathrm{RN}$ were effective. Control subjects showed declining levels of CRs during extinction sessions typical of tone-alone training $\left(F_{(3,12)}=7.04\right.$; $p<0.006)$. Release of the inactivations during extinction training (phase III) revealed no differences in CR frequencies between groups $\left(F_{(1,10)}=0.329 ; p=0.579\right)$. A significant day effect during phase III was found indicative of loss of performance with tonealone training $\left(F_{(3,30)}=3.89 ; p=0.018\right)$. During the last phase, animals in the experimental group were able to reacquire the association and showed response frequencies that were no different from controls $\left(F_{(1,10)}=0.22 ; p=0.87\right)$.

\section{Discussion}

Inactivations of the IN completely prevent extinction of the response yet do not interfere with the ability to extinguish the CR once inactivations are removed. Furthermore, although inactivations of both the IN and RN completely block response expression, only IN inactivations result in complete blockage of extinction. These observations were further explored by experimentally maintaining the behavioral response during IN inactivation. Data from experiment 2 clearly showed robust extinction immediately after release of IN inactivation, indicating that stimulations of the RN can remove the effects of blockage of extinction caused by functional disruption of the IN.

The fact that stimulations of the RN can remove the effects of blockage of extinction caused by functional inactivation of the IN suggests the existence of an alternative pathway via the $\mathrm{RN}$ for extinction. A large body of evidence has demonstrated the essential role of the cerebellum in the acquisition of the response (Christian and Thompson, 2003), and several lines of evidence implicate the cerebellar cortex and IN in extinction of the CR (Perret and Mauk, 1995; Gould and Steinmetz, 1996; Ramnani and Yeo, 1996). Stimulation of the RN would have to act on a target that is not only capable of providing a "teaching signal" for extinction to the cerebellum but to one that received inputs from both the IN and the RN. A wealth of evidence supports the view that the inferior olive-climbing fiber projection system to the cerebellum 
is the essential teaching or reinforcing system for learning of the eyeblink CR (McCormick et al., 1985; Mauk et al., 1986; Thompson, 1988; Steinmetz et al., 1989; Kim et al., 1998; Thompson et al., 1998; Christian and Thompson, 2003). By the same token, this system could also play a key role in extinction of the CR.

The RN sends a GABAergic projection to the IO (Kennedy, 1979). Furthermore, electrical stimulation of the magnocellular $\mathrm{RN}$ results in marked inhibition of cells in the IO responsive to peripheral stimuli (Weiss et al., 1990; Horn et al., 1998), confirming the existence of RN-mediated IO inhibition using stimulation of the ventral funiculus of the spinal cord instead of peripheral stimulation. It is very possible that direct stimulation of the RN strongly inhibited activity within the IO, promoting changes responsible for extinction. Consistent with this explanation are data showing that inhibition of climbing fibers below equilibrium $(1 \mathrm{~Hz})$ provides the teaching signal that drives extinction (Medina et al., 2002). To our knowledge, this result was first predicted in a computational model of the cerebellar circuit by Allen et al. (2000). Activity of the IO below baseline levels would happen under conditions in which inhibition to this structure is not counteracted by excitatory input (i.e., air puff) such as in tone-alone extinction training. Although the main source of this inhibition has been shown to be the IN (Nelson et al., 1989; Kim et al., 1998), the strong $\mathrm{RN}$ stimulation applied in this experiment could have resulted in an inhibitory input from the $\mathrm{RN}$ that was sufficient to tilt the balance of climbing fibers below background levels.

Can this signal, conveyed by climbing fibers, modulate changes in the cerebellum? More importantly, can these changes result in extinction? Electrophysiological studies support the hypothesis that olivary activity can greatly influence patterns of cell firing and strength of inputs in the cerebellum. Rawson and Tilokskulchai (1981) showed that high rates of climbing fiber firing can completely inhibit Purkinje cell simple spikes. A different effect has been reported under conditions of low-rate climbing fiber discharge whereby Purkinje cell sensitivity to peripheral input is heightened after a complex spike (Ebner and Bloedel, 1981). This suggests that olivary activity may also importantly determine mossy fiber influence onto the cerebellar cortex. Indeed, coactivation of mossy and climbing fiber inputs induces long-term depression at parallel-Purkinje cell synapses (Ito, 1989; Linden and Connor, 1993) and also induces conditioned responses (Steinmetz et al., 1989), whereas activation of mossy fibers in the absence of climbing fiber input (such as in extinction training) leads to long-term potentiation (LTP) (Sakurai, 1987; Hansel et al., 2001). During tone-alone training, LTP would increase Purkinje cell input into the interpositus, restoring inhibitory activity to the IN and resulting in suppression of the CR .

Altogether with previous data, the present findings support the idea of an important inhibitory IN-IO signal conveyed during extinction. As found in experiment 3 , it can be proposed that inactivation of the $\mathrm{RN}$ does not block extinction because it does not affect IN-IO inhibition. Conversely, as was observed during IN inactivations, disrupting function of this nucleus would have blocked inhibitory input to the IO, effectively preventing extinction from occurring. Results of experiment 2 can thus be reconciled by proposing that "restoring" GABAergic input to the IO during extinction via the $\mathrm{RN}-\mathrm{IO}$ inhibitory pathway can create an effective substitution of an "extinction signal" normally conveyed by the IN (Fig. 6). It should be noted, however, that because the IO was not studied directly in this study, data here only provide support for the IO hypothesis and do not show direct evidence of its validity. Nonetheless, based on previous findings and the results of data presented here, it appears that, under nonex-

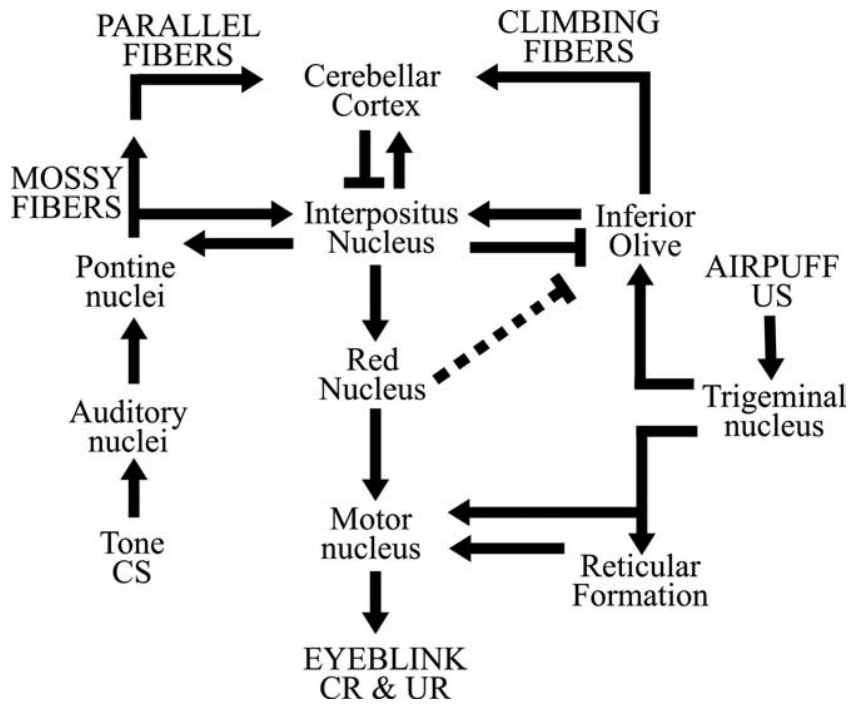

Figure 6. Highly simplified schematic of the cerebellum and associated brain circuitry essential for eyeblink conditioning. Note inhibitory connections from the IN to the I0. In extinction, IN inhibition would promote 10 activity decreases below baseline levels considering that the omission of the US would cease to excite climbing fibers. The strong IN inhibitory output that results during performance of the conditioned response without a US would then cause 10 activity to decrease, signaling changes that would ultimately allow extinction to occur. Dashed line indicates inhibitory rubro-olivary pathway activated during RN stimulation. This pathway is hypothesized to have provided an efficient substitution for a signal of extinction normally conveyed by the interpositus.

perimental conditions, the IN is the main source of this inhibitory input and might therefore be importantly involved in the induction of extinction.

Given the open debate regarding the validity of existing behavioral theories of extinction, findings of experiment 2 suggest that extinction occurred mainly because of the presence of CRs elicited through brain stimulation. Supporting response-extinction theories, this explanation of the results would also lead to the conclusion that IN inactivations block extinction simply because response expression is blocked. This interpretation was countered by findings of experiment 3 . Indeed, just as with IN inactivations, response expression was completely blocked during inactivation of the RN yet extinction occurred just as with normal control animals. Only blocking IN activity prevented extinction of the response. These results suggest that the IN is indeed a part of the essential neural circuit underlying extinction of the CR and that extinction might not involve mechanisms that rely on response performance [for similar findings using different learning paradigms (Schwaerzel et al., 2002; Ouyang and Thomas, 2005)].

Although data presented here do not speak directly to the context theory, they are at least not contradictory to it. Actually, extinction of the conditioned eyeblink response, unlike acquisition, is very dependent on context. Kehoe et al. (2004) and Poulos et al. (2006) established "extinction" of the CR simply by exposure to the conditioning context in the absence of CS presentations. Results of these behavioral studies on eyeblink conditioning as well as other learning paradigms (see above) support Bouton's (2004) comprehensive theory of extinction. As Bouton points out, the study by Krupa and Thompson (2003) showing that inactivation of the motor nuclei prevents CR performance and prevents extinction of the CR can be reinterpreted: animals received extinction training in a context (total paralysis of the ipsilateral facial musculature) that was very different from the training context. Indeed, inactivation of the $\mathrm{MN}$ not only results 
in severe motor deficits but also prevents response expression completely, whereas inactivation of the IN and RN does not; animals are still capable of spontaneous and reflexive blinks and show no overt drug side effects. The major change in context is that, in the MN inactivation study, the animal cannot move its face or ear, let alone blink, a much more pronounced change and a condition that predisposes to the "renewal" effect (Bouton and King, 1983). However, the neural bases of these possible context effects remain to be explored.

\section{References}

Allen MT, Myers CE, Gluck MA (2000) Neural network approaches to eyeblink classical conditioning. In: Eyeblink classical conditioning: animal (Woodruff-Pak DS, Steinmetz JE, eds), pp 229-255. Boston: Kluwer Academic.

Bouton ME (2004) Context and behavioral processes in extinction. Learn Mem 11:485-494.

Bouton ME, King DA (1983) Contextual control of the extinction of conditioned fear: tests for the associative value of the context. J Exp Psychol Anim Behav Process 9:248-265.

Bouton ME, Nelson JB (1994) Context-specificity of target versus feature inhibition in a feature-negative discrimination. J Exp Psychol Anim Behav Process 20:51-65.

Chapman PF, Steinmetz JE, Thompson RF (1988) Classical conditioning does not occur when direct stimulation of the red nucleus or cerebellar nuclei is the conditioned stimulus. Brain Res 442:97-104.

Christian KM, Thompson RF (2003) Neural substrates of eyeblink conditioning: acquisition and retention. Learn Mem 10:427-455.

Clark RE, Lavond DG (1993) Reversible lesions of the red nucleus during acquisition and retention of a classically conditioned behavior in rabbits. Behav Neurosci 107:264-270.

Ebner TJ, Bloedel JR (1981) Role of climbing fiber afferent input in determining responsiveness of Purkinje cells to mossy fiber inputs. J Neurophysiol 45:962-971.

Gould TJ, Steinmetz JE (1996) Changes in rabbit cerebellar cortical and interpositus nucleus activity during acquisition, extinction, and backward classical eyelid conditioning. Neurobiol Learn Mem 65:17-34.

Hansel C, Linden DJ, D’Angelo E (2001) Beyond parallel fiber LTD: the diversity of synaptic and non-synaptic plasticity in the cerebellum. Nat Neurosci 4:467-475.

Hardiman MJ, Ramnani N, Yeo CH (1996) Reversible inactivations of the cerebellum with muscimol prevent the acquisition and extinction of conditioned nictitating membrane responses in the rabbit. Exp Brain Res 110:235-247.

Horn KM, Hamm TM, Gibson AR (1998) Red nucleus stimulation inhibits within the inferior olive. J Neurophysiol 80:3127-3136.

Ito M (1989) Long-term depression. Annu Rev Neurosci 12:85-102.

Kehoe EJ, Weidemann G, Dartnall S (2004) Apparatus exposure produces profound declines in conditioned nictitating-membrane responses to discrete conditioned stimuli by the rabbit (Oryctalagus cuniculus). J Exp Psychol Anim Behav Process 30:259-270.

Kennedy PR (1979) The rubro-olivo-cerebellar teaching circuit. Med Hypotheses 5:799-807.

Kim JJ, Krupa DJ, Thompson RF (1998) Inhibitory cerebello-olivary projections and blocking effect in classical conditioning. Science 279:570-573.

Krupa DJ (1993) Localization of the essential memory trace for a classically conditioned behavior. PhD thesis, University of Southern California.

Krupa DJ, Thompson RF (1997) Reversible inactivation of the cerebellar interpositus nucleus completely prevents acquisition of the classically conditioned eye-blink response. Learn Mem 3:545-556.

Krupa DJ, Thompson RF (2003) Inhibiting the expression of a classically conditioned behavior prevents its extinction. J Neurosci 23:10577-10584.

Krupa DJ, Thompson JK, Thompson RF (1993) Localization of a memory trace in the mammalian brain. Science 260:989-991.

Krupa DJ, Weng J, Thompson RF (1996) Inactivation of brainstem motor nuclei blocks expression but not acquisition of the rabbit's classically conditioned eyeblink response. Behav Neurosci 110:219-227.

Lavond DG, Steinmetz E (1989) An inexpensive interface for the IBM PC/XT and compatibles. Behav Res Methods 21:435-440.

Linden DJ, Connor JA (1993) Cellular mechanisms of long-term depression in the cerebellum. Curr Opin Neurobiol 3:401-406.
McBride RL, Klemm WR (1968) Stereotaxic atlas of rabbit brain, based on the rapid method of photography of frozen, unstained sections. Commun Behav Biol 2:179-215.

McCormick DA, Steinmetz JE, Thompson RF (1985) Lesions of the inferior olivary complex cause extinction of the classically conditioned eyeblink response. Brain Res 359:120-130.

Mauk MD, Ohyama T (2004) Extinction as new learning versus unlearning: considerations from a computer simulation of the cerebellum. Learn Mem 11:566-571.

Mauk MD, SteinmetzJE, Thompson RF (1986) Classical conditioning using stimulation of the inferior olive as the unconditional stimulus. Proc Natl Acad Sci USA 83:5349-5353.

Medina JF, Nores WL, Mauk MD (2002) Inhibition of climbing fibres is a signal for the extinction of conditioned eyelid responses. Nature 416:330-333.

Nelson BJ, Adams JC, Barmack NH, Mugnaini E (1989) Comparative study of glutamate decarboxylase immunoreactive boutons in the mammalian inferior olive. J Comp Neurol 286:514-539.

Nores WL, Medina JF, Steele PM, Mauk MM (2000) Relative contributions of the cerebellar cortex and cerebellar nucleus to eyelid conditioning. In: Eyeblink classical conditioning: animal models (Woodruff-Pak DS, Steinmetz JE, eds), pp 205-228. Boston: Kluwer Academic.

Nowak AJ, Marshall-Goodell B, Kehoe EJ, Gormezano I (1997) Elicitation, modification, and conditioning of the rabbit nictitating membrane response by electrical stimulation in the spinal trigeminal nucleus, inferior olive, interpositus nucleus, and red nucleus. Behav Neurosci 111:1041-1055.

Ouyang M, Thomas SA (2005) A requirement for memory retrieval during and after long-term extinction learning. Proc Natl Acad Sci USA 102:9347-9352.

Perrett SP, Mauk MD (1995) Extinction of conditioned eyelid responses requires the anterior lobe of cerebellar cortex. J Neurosci 15:2074-2080.

Poulos AM, Pakaprot N, Mahdi B, Kehoe EJ, Thompson RF (2006) Decremental effects of context exposure following delay eyeblink conditioning in rabbits. Behav Neurosci 120:730-734.

Ramnani N, Yeo CH (1996) Reversible inactivations of the cerebellum prevent the extinction of conditioned nictitating membrane responses in rabbits. J Physiol (Lond) 495:159-168.

Rawson JA, Tilokskulchai K (1981) Repetitive firing of cerebellar Purkinje cells in response to impulse in climbing fibre afferents. Neurosci Lett 25:131-135.

Rescorla RA (1997) Response-inhibition in extinction. Q J Exp Psychol $50: 238-252$.

Rescorla RA (2004) Spontaneous recovery. Learn Mem 11:501-509.

Robleto K, Poulos AM, Thompson RF (2004) Brain mechanisms of extinction of the classically conditioned eyeblink response. Learn Mem 11:517-524.

Sakurai M (1987) Synaptic modification of parallel fibre-Purkinje cell transmission in in vitro guinea-pig cerebellar slices. J Physiol (Lond) 394:463-480.

Schwaerzel M, Heisenberg M, Zars T (2002) Extinction antagonizes olfactory memory at the subcellular level. Neuron 35:951-960.

Steinmetz JE (2000) Brain substrates of classical eyeblink conditioning: a highly localized but also distributed system. Behav Brain Res 110:13-24.

Steinmetz JE, Lavond DG, Thompson RF (1989) Classical conditioning in rabbits using pontine nucleus stimulation as a conditioned stimulus and inferior olive stimulation as an unconditioned stimulus. Synapse 3:225-233.

Thomas SA, Marck BT, Palmiter RD, Matsumoto AM (1998) Restoration of norepinephrine and reversal of phenotypes in mice lacking dopamine beta-hydroxylase. J Neurochem 70:2468-2476.

Thompson RF (1988) The neural basis of basic associative learning of discrete behavioral responses. Trends Neurosci 11:152-155.

Thompson RF, Krupa DJ (1994) Organization of memory traces in the mammalian brain. Annu Rev Neurosci 17:519-549.

Thompson RF, Thompson JK, Kim JJ, Krupa DJ, Shinkman PG (1998) The nature of reinforcement in cerebellar learning. Neurobiol Learn Mem 70:150-176.

Weiss C, Houk JC, Gibson AR (1990) Inhibition of sensory responses of cat inferior olive neurons produced by stimulation of red nucleus. J Neurophysiol 64:1170-1185.

Yeo CH, Hesslow G (1998) Cerebellum and conditioned reflexes. Trends Cogn Sci 2:322-330. 